\title{
Implications of IDH mutations on immunotherapeutic strategies for malignant glioma
}

\author{
Leland G. Richardson, MMSc, MS,, 2 Julie J. Miller, MD, PhD, ${ }^{3}$ Yosuke Kitagawa, MD, PhD, ${ }^{2}$ \\ Hiroaki Wakimoto, MD, PhD, ${ }^{2}$ Bryan D. Choi, MD, PhD, ${ }^{1,2}$ and William T. Curry, MD ${ }^{1,2}$ \\ ${ }^{1}$ Translational Brain Tumor Immunology Laboratory, Massachusetts General Hospital, Harvard Medical School, Boston, \\ Massachusetts; '2Department of Neurosurgery, Massachusetts General Hospital, Harvard Medical School, Boston, \\ Massachusetts; and ${ }^{3}$ Pappas Center for Neuro-Oncology, Department of Neurology, Massachusetts General Hospital, Harvard \\ Medical School, Boston, Massachusetts
}

\begin{abstract}
Immunotherapy has emerged as a promising approach for treating aggressive solid tumors, even within the CNS. Mutation in the metabolic gene isocitrate dehydrogenase 1 (IDH1) represents not only a major glioma defining biomarker but also an attractive therapeutic neoantigen. As patients with IDH-mutant glioma enter early-phase vaccine and immune checkpoint inhibitor clinical trials, there is emerging evidence that implicates the oncometabolite, 2-hydroxyglutarate $(2 \mathrm{HG})$, generated by the neomorphic activity of mutant IDH, as a potential barrier to current immunotherapeutic approaches. Here, the authors review the immunomodulatory and immunosuppressive roles of $2 \mathrm{HG}$ within the unique IDHmutant glioma tumor immune microenvironment and discuss promising immunotherapeutic approaches currently being investigated in preclinical models.
\end{abstract}

https://thejns.org/doi/abs/10.3171/2021.11.FOCUS21604

KEYWORDS glioma; isocitrate dehydrogenase; 2-hydroxyglutarate; immunosuppression; tumor immune microenvironment; IDH; immunology

$\mathrm{G}$ LIOMAS represent the most common primary brain tumor of the CNS. Resection, radiation therapy, and chemotherapy remain the standard treatment. Isocitrate dehydrogenase 1 or 2 (IDH1 and IDH2, respectively) mutations are frequently expressed in historically defined grade II and III gliomas. ${ }^{1}$ Gliomas with IDH mutations display a more indolent disease course and favorable prognosis compared with their wild-type counterparts; however, these tumors usually recur. Considering that mutant IDH is an early oncogenic driver as well as a neoantigen found exclusively and uniformly in nearly all tumor cells and otherwise absent in normal tissues, the IDH mutation represents an ideal therapeutic target. ${ }^{2}$

Immunotherapy is at the forefront of cancer treatment, ${ }^{3,4}$ and several immune-based treatment strategies have entered early-phase clinical trials for IDH-mutant glioma, including the targeting of mutant IDH with a neo- antigen peptide vaccine. ${ }^{5}$ In this review, we focus on the emerging data describing the unique immune landscape of IDH-mutant glioma and the role of 2-hydroxyglutarate $(2 \mathrm{HG})$ as a regulator of glioma immune evasion. We also discuss novel immunotherapeutic strategies on the horizon for patients with IDH-mutant glioma.

\section{Immunotherapy for IDH-Mutant Glioma: Clinical Studies \\ IDH Peptide Vaccination}

Three different IDH1-R132H-specific peptide vaccines are currently being tested in 4 clinical trials (Table 1 ), one of which recently released results (ClinicalTrials. gov identifier no. NCT02454634). In 2021, Platten and colleagues reported a phase I trial showing that a 20-amino acid peptide vaccine spanning the mutated region was ca-

ABBREVIATIONS AhR = aryl hydrocarbon receptor; $\mathrm{GBM}=$ glioblastoma; $\mathrm{ICI}=$ immune checkpoint inhibitor; IDH = isocitrate dehydrogenase; $\mathrm{IHC}=$ immunohistochemistry; IL-10 = interleukin-10; MDM = monocyte-derived macrophage; $M$ G = tumor resident microglia; NK = natural killer; PD-1 = programmed death-1; PD-L1 = programmed death ligand-1; TCR = T-cell receptor; TIME = tumor immune microenvironment; TMB = tumor mutational burden; Treg = regulatory T cell; $2 \mathrm{HG}=2$-hydroxyglutarate. SUBMITTED October 7, 2021. ACCEPTED November 19, 2021.

INCLUDE WHEN CITING DOI: 10.3171/2021.11.FOCUS21604. 
TABLE 1. Overview of immunotherapy clinical trials exclusively in IDH-mutant glioma patients

\begin{tabular}{|c|c|c|c|c|c|c|c|c|}
\hline & Registry No. & Institution & Immunotherapy & $\mathrm{IDHi}$ & Setting & Phase & Treatment Regimen & Status \\
\hline \multirow{3}{*}{ Vaccine } & NCT02454634 & $\begin{array}{l}\text { German Cancer } \\
\quad \text { Research Center }\end{array}$ & IDH1 vaccine & No & Primary & I & $\begin{array}{l}\text { IDH1 vaccine } \pm \mathrm{RT} \pm \\
\quad \mathrm{TMZ}\end{array}$ & Completed \\
\hline & NCT02193347 & $\begin{array}{l}\text { Duke University Medical } \\
\text { Center }\end{array}$ & PEPIDH1M & No & Recurrent & 1 & PEPIDH1M \pm RT + TMZ & $\begin{array}{l}\text { Active, not } \\
\text { recruiting }\end{array}$ \\
\hline & NCT02771301 & Beijing Tiantan Hospital & $\begin{array}{l}\text { IDH1R132H- } \\
\text { DC vaccine }\end{array}$ & No & Primary & I & $\begin{array}{l}\text { IDH1R132H-DC vaccine } \\
\quad+\mathrm{RT}+\mathrm{TMZ}\end{array}$ & Recruiting \\
\hline $\begin{array}{l}\text { Vaccine + checkpoint } \\
\text { inhibitor }\end{array}$ & NCT03893903 & $\begin{array}{l}\text { German Cancer } \\
\text { Research Center }\end{array}$ & $\begin{array}{l}\text { IDH1-vac + } \\
\text { anti-PD-L1 }\end{array}$ & No & Recurrent & I & $\begin{array}{l}\text { IDH1 vaccine } \pm \text { avelu- } \\
\text { mab }\end{array}$ & Recruiting \\
\hline \multirow{6}{*}{ Checkpoint inhibitor } & NCT03991832 & $\begin{array}{l}\text { University Health } \\
\text { Network, Toronto }\end{array}$ & Anti-PD-L1 & No & Recurrent & II & $\begin{array}{l}\text { Durvalumab + olaparib } \\
\text { (PARPi) }\end{array}$ & Recruiting \\
\hline & NCT03925246 & Hôpitaux de Paris & Anti-PD-1 & No & Recurrent & $\|$ & Nivolumab & $\begin{array}{l}\text { Active, not } \\
\text { recruiting }\end{array}$ \\
\hline & NCT04056910 & $\begin{array}{l}\text { University of Pittsburgh } \\
\text { Medical Center }\end{array}$ & Anti-PD-1 & Yes & Recurrent & II & Nivolumab + ivosidenib & Recruiting \\
\hline & NCT02968940 & NYU Langone Health & Anti-PD-L1 & No & Recurrent & II & Avelumab + HFRT & Completed \\
\hline & NCT03718767 & $\begin{array}{l}\text { National Institutes of } \\
\text { Health Clinical Center }\end{array}$ & Anti-PD-1 & No & Recurrent & II & Nivolumab & Recruiting \\
\hline & NCT03557359 & $\begin{array}{l}\text { Columbia University } \\
\text { Medical Center }\end{array}$ & Anti-PD-1 & No & Recurrent & II & Nivolumab & Recruiting \\
\hline
\end{tabular}

HFRT = hypofractionated radiotherapy; IDHi = IDH inhibitor; PARPi = poly(ADP-ribose) polymerase inhibitor; RT = radiotherapy; TMZ = temozolomide.

pable of inducing T-cell responses in patients with newly diagnosed WHO grade III and IV IDH1-R132H-positive gliomas. ${ }^{5}$ Of the 44 patients initially enrolled in the study, 33 patients received 8 vaccines over a period of 6 months, integrated with radiotherapy and adjuvant temozolomide regimens. The treatment was safe, with no severe treatment-related adverse events. Although the trial was not powered to demonstrate clinical efficacy, several biologically important findings were noted. First, there was a strong positive correlation between IDH1-R132H peptide presentation in the tumor tissue and vaccine-induced peripheral T-cell responses. Also, the presence of pseudoprogression after vaccine administration was associated with an increased number of vaccine-induced mutant IDH1-specific T cells infiltrating the tumor, suggestive of intratumoral inflammatory responses. Overall, these results demonstrate that the IDH1-R132H peptide vaccine may elicit specific immune responses. Currently, this same group is testing the safety and immunogenicity of combining immune checkpoint inhibitors (ICIs) with the same peptide vaccine in a separate randomized phase I study (ClinicalTrials.gov identifier no. NCT03893903).

\section{Immune Checkpoint Inhibition}

Currently, there are a total of 6 ongoing clinical trials targeting programmed death-1 (PD-1) or programmed death ligand-1 (PD-L1) exclusively in patients with recurrent IDH-mutant glioma (Table 1). There is a relative dearth of knowledge surrounding the responsiveness of IDH-mutant tumors to ICI since, to date, most brain tumor clinical trial data reflect activity in glioblastoma (GBM), IDH-wild-type disease. ${ }^{6,7}$ Despite the recent negative phase III results in GBM from the ICI "CheckMate" trials,
IDH-mutant glioma patients have entered the ICI space. The rationale for the use of ICIs in patients with recurrent IDH-mutant glioma is based on data suggesting that alkylating agents such as temozolomide and lomustine promote the emergence of a hypermutated phenotype.$^{8,9}$ Heavily treated glial tumors often reach a hypermutant phenotype,$^{10}$ and the resulting high tumor mutational burden (TMB) may improve responsiveness to ICI. ${ }^{7}$ Of the 6 trials, 4 trials are currently recruiting, 1 is active, and 1 is complete. The recently completed phase II trial (ClinicalTrials.gov identifier no. NCT02968940) investigated the concurrent administration of a PD-L1 inhibitor (avelumab) with hypofractionated radiation therapy in patients with recurrent grade IV IDH-mutant glioma.

\section{The Immune Landscape of Mutant IDH: A Brief Overview}

The tumor immune microenvironment (TIME) in glioma is a complex milieu of various noncancerous cell types and components that include infiltrating immune cells, fibroblasts, endothelial cells, and extracellular matrix. ${ }^{11}$ It is well appreciated that the immune contexture, specifically the immune cell type, density, localization, orientation, and functional status, all play a significant role in disease progression and influence the efficacy of immune-based therapies. ${ }^{12}$ Tumor immunology in the brain is unique. Bulk sequencing studies comparing the host immune responses across various cancer types have defined gliomas as "lymphocyte depleted" and/or "immunologically quiet" tumors. ${ }^{13}$ Since the prioritization of molecular features in the classification of gliomas, ${ }^{1}$ growing evidence suggests that IDH mutations influence this immunologically "cold" 
tumor phenotype. ${ }^{14}$ Recent advances in high-dimensional immune profiling techniques such as single-cell RNA sequencing, mass cytometry, and multiplexed immunohistochemistry (IHC) have allowed researchers to gain an even more granular picture of the similarities and differences of the immune contexture between IDH-mutant and IDHwild-type gliomas.

Several studies have demonstrated that IDH mutations in glioma are associated with less infiltration of CD3+, CD4+, and CD8+ T cells in comparison with their wildtype counterparts..$^{15,16}$ In addition to effector T-cell subsets, our group recently showed a significant reduction in suppressive regulatory T cells (Tregs) in IDH-mutant tumors. ${ }^{17}$ In an investigation of a large cohort of diffuse glioma, Berghoff and colleagues showed that the expression of PD-L1, an immunosuppressive cell surface molecule that downregulates T-cell activity, was significantly reduced in IDH-mutant tumors. ${ }^{16}$ Recent work by Friebel et al. and Mathewson et al. further interrogated the transcriptional profiles of tumor-infiltrating T cells and compared their activation states between IDH mutational status. ${ }^{18,19}$ Their work demonstrated stronger antitumor immunity in IDH-wild-type GBM compared with IDHmutant glioma based on single-cell profiling of CD8+ and CD4+ T cells and their respective cytotoxicity/interferon signatures. Given the potential predictive value of tumor-infiltrating lymphocytes and PD-L1 expression for responsiveness to ICI, these data suggest that untreated tumors harboring IDH mutations are inferior responders to ICI compared with IDH-wild-type gliomas based on their "colder" phenotype.

In a separate study focusing on CD68+ tumor-associated macrophages/microglia, Klemm et al. used multiplexed IHC to reveal a greater proportion of tumorresident microglia (MG) in IDH-mutant tumors, whereas IDH-wild-type tumors were more heavily infiltrated with monocyte-derived macrophages (MDMs). ${ }^{20}$ In addition, single-cell profiling of both MG and MDM populations demonstrated a more complex and multifaceted phenotype than the classic M1 versus M2 phenotype, with distinct transcriptional signatures that largely aligned with the tumor's IDH mutational status. Infiltration of myeloid cells, which are predominantly immunosuppressive, is generally linked to a poorer prognosis in glioma. ${ }^{21} \mathrm{IDH}-$ mutant tumors exhibit less myeloid cell infiltration compared with their wild-type counterpart, which may explain their more indolent disease course; ${ }^{22}$ however, there is no evidence to point specifically to this hypothesis. The distorted immune infiltrate, activation states, and overall tumor immunogenicity unique to mutant disease may play a role in driving response to standard therapy and their prognostic profile. ${ }^{23}$ Growing knowledge of the specific characteristics of the IDH-mutant TIME may help define a more delineated immune modulatory approach for this subset of patients.

Differences in the immune cell composition of IDHmutant tumors may be largely driven by the accumulation of the oncometabolite, $2 \mathrm{HG}$, produced by the neomorphic activity of the mutant IDH enzyme. ${ }^{14} 2 \mathrm{HG}$ has been implicated in reprogramming the glioma cell transcriptome by competitively inhibiting key $\alpha$-ketoglutarate-depen- dent histone demethylase enzymes. ${ }^{24}$ This leads to a hypermethylated phenotype within IDH-mutant tumor cells, which, in turn, promotes the global repression of genes, including those involved in the immune system. ${ }^{22}$ Using human samples and preclinical glioma models, both Amankulor et al. and Kohanbash et al. have demonstrated that glioma cells expressing mutant IDH downregulate an array of genes associated with leukocyte recruitment, resulting in decreased intratumoral immune cell infiltration. ${ }^{15,22}$ This diminished immune phenotype exhibited by IDH-mutant tumors has been consistently recapitulated in human samples and by other groups studying the immunological consequence of mutant IDH. ${ }^{16,17,19,20,25}$ The distinctive immune milieu in IDH-mutant tumors (Fig. 1) may be relevant when developing immune modulating strategies in the future for this molecular class of patients.

\section{Immunosuppressive Effects of 2HG}

The accumulation of $2 \mathrm{HG}$ has long been shown to reprogram the cellular metabolism, epigenetics, and oncogenic processes in malignant glioma. ${ }^{24}$ Several groups have directed their attention to the impact of the oncometabolite on antitumor immune responses.

\section{HG-Mediated "Indirect" Immunosuppression}

$2 \mathrm{HG}$ prevents the recruitment of $\mathrm{CD} 8+\mathrm{T}$ cells through a tumor intrinsic mechanism. By introducing mutant IDH into a murine glioma cell line or treating parental wildtype cell lines with $2 \mathrm{HG}$, Kohanbash et al. observed a marked reduction in the expression of cytotoxic $\mathrm{T}$ lymphocyte-associated genes, interferon- $\gamma$-inducible chemokines, including CXC-chemokine ligand $10 .{ }^{15}$ Moreover, signal transducer and activator of transcription 1, a key regulator of these genes, was also repressed in IDH-mutant cells compared with IDH-wild-type cells. Mice injected with syngeneic mouse glioma harboring the IDH mutation demonstrated reduced CD8+ T-cell infiltration and lower levels of CXC-chemokine ligand 10 in their tumors. The use of an inhibitor of $2 \mathrm{HG}$ reversed the immune deficits, resulting in an increase in T-cell recruitment to the tumor in mice bearing IDH-mutant glioma..$^{15}$

In addition to its impact on CD8+ T-cell recruitment, Zhang et al. demonstrated another tumor intrinsic alteration driven by IDH mutations that resulted in the escape of natural killer (NK) cell-mediated killing. ${ }^{26}$ In a similar experimental design, they showed that $2 \mathrm{HG}$ was directly responsible for driving the repression of activating receptor NKG2D ligands ULBP1 and ULBP3 genes, leading to IDH-mutant cell resistance to NK cells. Decitabine, a hypomethylating agent, was shown to increase the expression of ULBP1 and ULBP3, restoring NK cell-mediated lysis of IDH-mutant cells.

\section{HG-Mediated "Direct" Immunosuppression}

2HG has been shown to directly suppress the activation and proliferation of $\mathrm{T}$ cells. ${ }^{25}$ Bunse et al. demonstrated that $2 \mathrm{HG}$ can be taken up by $\mathrm{T}$ cells and impair T-cell receptor (TCR) signaling and polyamine biosynthesis, which resulted in the suppression of nuclear factor of activated T-cell activity, a critical transcription factor that 


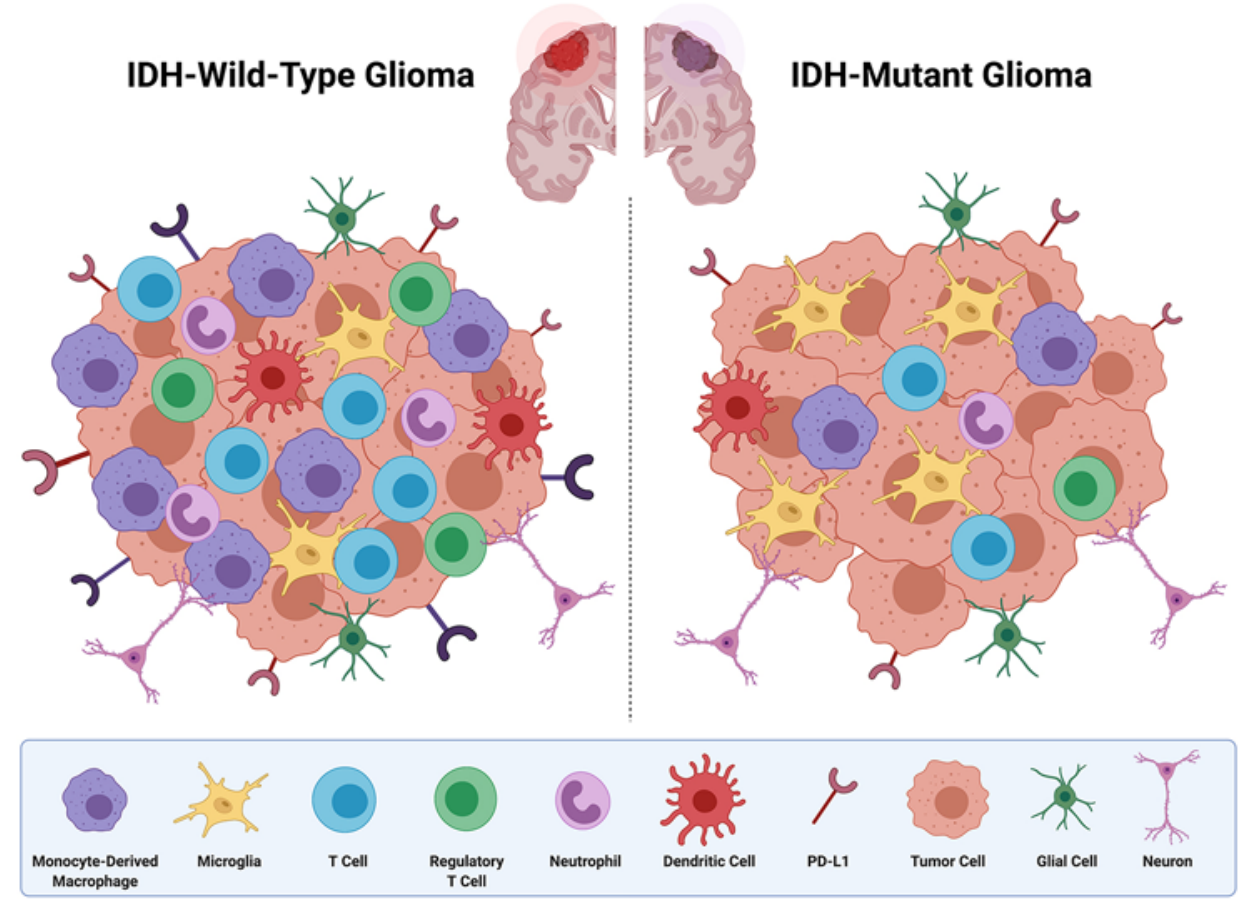

FIG. 1. Immune landscape of IDH-mutant glioma. The relative frequency and actionable targets of infiltrating immune cells are important points to consider when designing an immunotherapeutic approach. The major immune cell populations infiltrating glioma differ significantly between IDH mutational status. IDH-mutant glioma is characterized as immunologically quieter than its wild-type counterpart. The predominant immune cells infiltrating IDH-mutant glioma are MG followed by MDMs. T cells are very low in abundance in IDH-mutant glioma. This distorted immune composition is largely driven by the oncometabolite $2 \mathrm{HG}$. Created with BioRender.com.

activates T-cell effector functions. ${ }^{25}$ With the use of an IDH inhibitor in various tumor models, the authors were able to demonstrate that $2 \mathrm{HG}$ dampened antitumor T-cell immunity induced by IDH1-R132H peptide vaccination and PD-1 inhibition. Infiltrating MDMs were also paracrine targets of $2 \mathrm{HG}$. Friedrich et al. demonstrated that $2 \mathrm{HG}$ reprogrammed MDMs toward an immunosuppressive phenotype by altering tryptophan-related metabolic pathways. ${ }^{27} 2 \mathrm{HG}$ led to the increased uptake/degradation of L-tryptophan and activity of cytoplasmic aryl hydrocarbon receptor (AhR), a key transcription factor that is known to polarize MDMs toward an immunosuppressive phenotype. This work showed that $2 \mathrm{HG}$ caused the increased activation of AhR signaling, which resulted in the elevated secretion of interleukin-10 (IL-10) and transforming growth factor- $\beta$, two known immunosuppressive molecules that can decrease T-cell activation and proliferation. Furthermore, $2 \mathrm{HG}$ also lowered the expression of costimulatory molecules CD80 and CD86 and major histocompatibility complex class II (MHC-II), which reduced the capacity for optimal antigen presentation further driving a tolerogenic phenotype in MDMs. The authors also noted that $2 \mathrm{HG}$-induced T-cell suppression required both the 2HG-mediated uptake of L-tryptophan and functioning AhR in MDMs. While an IDH inhibitor partially restored MDM antigen presentation in mice bearing IDHmutant tumors, the use of an AhR inhibitor significantly reversed the $2 \mathrm{HG}$-mediated immunosuppressive pheno- type in MDMs. ${ }^{27}$ These data not only demonstrate a dual immunomodulatory mechanism of immune evasion mediated by the production of $2 \mathrm{HG}$ (Fig. 2) but also suggest that IDH-mutant gliomas are potentially more resistant to immunotherapeutic approaches.

\section{Mutant IDH Inhibition: Critical to the Immunotherapeutic Strategy?}

The studies above support the use of specific inhibitors of IDH to reverse $2 \mathrm{HG}$-mediated immune suppression, which may improve the efficacy of immunotherapy in patients with IDH-mutant gliomas. Since the discovery of IDH, much effort has been aimed at developing clinical-grade inhibitors specifically targeting mutant IDH1 or IDH2 with the belief that IDH-mutant gliomas are biologically reliant on $2 \mathrm{HG}$ as an oncogenic driver. Patients with acute myeloid leukemia and cholangiocarcinoma harboring mutant IDH have had objective responses, ${ }^{28,29}$ which has led to IDH-inhibitor approval from the US FDA. However, the efficacy of these inhibitors as a monotherapy in glioma patients remains unproven. With growing evidence that $2 \mathrm{HG}$ acts as an immunosuppressive molecule in glioma, perhaps the focus should shift from a monotherapy to uncovering the utility of inhibitors as a sensitizing agent for immune-based approaches for IDH-mutant tumors.

Promising data from an ongoing phase I clinical trial 

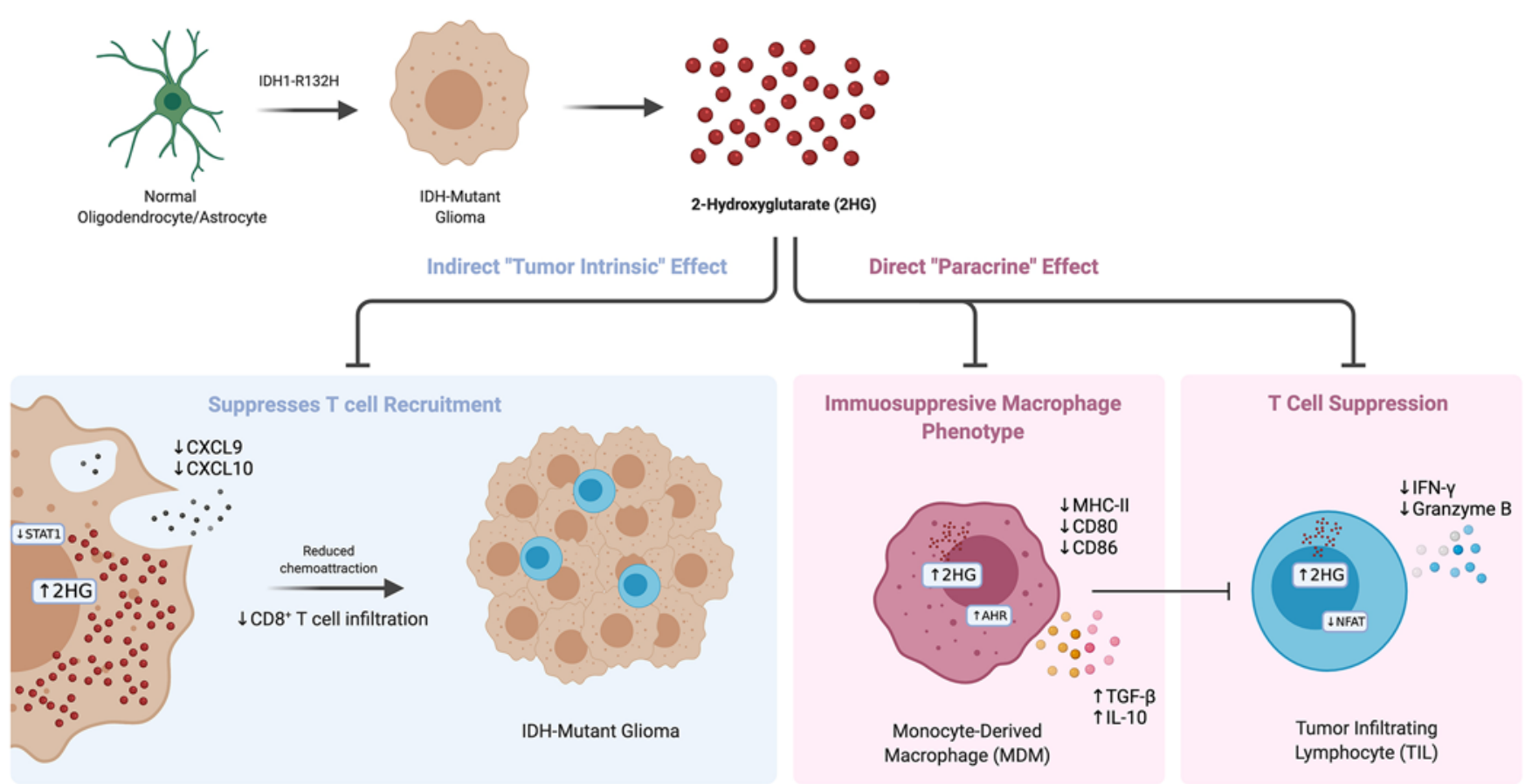

FIG. 2. 2HG suppresses antitumor T-cell responses through a direct and indirect mechanism. Antitumor T-cell immunity is not at full capacity within the IDH-mutant glioma microenvironment. The accumulation of intracellular $2 \mathrm{HG}$ generated by mutant IDH downregulates the production of chemokines that attract CD8+ T cells to the tumor. Moreover, $2 \mathrm{HG}$ can be exported out of the tumor cell and directly suppress effector T-cell function. The activity and proliferation of T cells are further suppressed by infiltrating MDMs that exhibit an immunosuppressive phenotype mediated by $2 \mathrm{HG}$. In preclinical models, IDH inhibition reinvigorates T-cell activity and reprograms the immunosuppressive myeloid phenotype to reverse $2 \mathrm{HG}$-mediated immune suppression. The use of an IDH inhibitor may sensitize IDH-mutant glioma to immune-based therapies. $\mathrm{CD}=$ cluster of differentiation; CXCL9 = CXC-chemokine ligand; $C X C L 10=C X C$-chemokine ligand 10; IFN- $y=$ interferon gamma; $\mathrm{MHC}$ - II = major histocompatibility complex II; STAT1 = signal transducer and activator of transcription 1 ; TGF- $\beta=$ transforming growth factor- $\beta$. Adapted from "Immune Suppressive Effects of TGF- $\beta$ in the Tumor Microenvironment," by Biorender.com (2021). Retrieved from https://app.biorender.com/biorender-templates.

(ClinicalTrials.gov identifier no. NCT03343197) in patients with recurrent low-grade gliomas reported at the 2020 American Association for Cancer Research Annual Meeting suggested that IDH inhibition with ivosidenib (AG-120) or vorasidenib (AG-881) reverses $2 \mathrm{HG}$ mediated immune suppression and may sensitize IDH-mutant gliomas for ICI therapy. ${ }^{30} \mathrm{Lu}$ et al. measured infiltrating $\mathrm{T}$ cells, MDMs/MG, and PD-L1 expression in paired preand posttreatment samples. Using IHC and TCR sequencing, they found that inhibition of mutant IDH increased the number of total CD3+ and CD8+ T cells, TCR clones, and TCR diversity. ${ }^{30}$ Using RNA sequencing, they also observed a polarization toward a proinflammatory tumorassociated macrophage/microglia signature as well as an increase in PD-L1 expression after treatment. These data are consistent with recently published preclinical data in which an increase in PD-L1 expression was observed in mice bearing IDH-mutant gliomas after IDH inhibition. ${ }^{31}$ Although these data support the idea that IDH inhibition may improve the efficacy of immune-based approaches for IDH-mutated glioma, additional studies are needed to validate these observations as well as address the concern of other barriers, such as immunosuppressive mediators and infiltrate. Of the active immunotherapy clinical trials for IDH-mutant glioma, a phase II study (ClinicalTrials. gov identifier no. NCT04056910) led by investigators at the University of Pittsburgh Medical Center is the only trial investigating the combination of a PD-1 inhibitor (nivolumab) with IDH inhibition (ivosidenib).

\section{Novel Immunotherapeutic Strategies for IDH-Mutant Gliomas: Preclinical Models}

The notion that IDH inhibition should be integrated into immunotherapy for patients with IDH-mutant glioma is based on preclinical studies that have demonstrated successful results incorporating an inhibitor with either vaccination or ICI in mice bearing IDH-mutant tumors (Table 2). To date, preclinical immunotherapeutic strategies that have incorporated IDH inhibition fall into two categories.

\section{Vaccine With IDH Inhibition}

Kohanbash et al. utilized a vaccine strategy using a combination of synthetic peptides derived from gliomaassociated antigens. ${ }^{15}$ They demonstrated that after vaccination, mice challenged with IDH-mutant tumors receiving daily treatment of an IDH inhibitor (IDH-35) survived 
TABLE 2. Overview of novel immune-based approaches with an IDH inhibitor in preclinical models

\begin{tabular}{|c|c|c|c|}
\hline Authors \& Year & Engineered IDH-Mutant Mouse Model & Treatment Regimen† & Outcome \\
\hline $\begin{array}{l}\text { Kadiyala et al., } \\
2021^{31}\end{array}$ & shp53/shATRX/mIDH1R132H & \pm Anti-PD-L1 \pm IR $\pm \mathrm{TMZ} \pm{ }^{*} \mathrm{AGI}-5198$ & $\begin{array}{l}\text { Complete tumor regression in } 60 \% \text { of mice when } \\
\text { IDHi was combined w/ SOC \& checkpoint } \\
\text { blockade; reversed immunosuppressive } \\
\text { phenotype of infiltrating myeloid cells }\end{array}$ \\
\hline $\begin{array}{l}\text { Bunse et al., } \\
2018^{25}\end{array}$ & GL261-transduced IDH1R132H & \pm Anti-PD-1 $\pm{ }^{*}$ BAY1436032 & $\begin{array}{l}\text { Enhanced efficacy of checkpoint inhibition w/ } \\
\text { IDHi; adoptive transfer of vaccine-induced T } \\
\text { cells reduced tumor growth only w/ IDHi }\end{array}$ \\
\hline $\begin{array}{l}\text { Kohanbash et al., } \\
2017^{15}\end{array}$ & GL261-transduced IDH12132H & \pm Peptide vaccine $\pm{ }^{*} \mathrm{IDH}-\mathrm{C} 35$ & $\begin{array}{l}\text { Enhanced efficacy of vaccine w/ IDHi; increased } \\
\text { intratumoral CD8+ T cells }\end{array}$ \\
\hline
\end{tabular}

$\mathrm{IR}=$ irradiation; $\mathrm{SOC}=$ standard of care; $\pm=$ with or without.

$\dagger$ Drug names following the asterisks denote the particular IDH inhibitor used in the study.

significantly longer than mice with IDH-mutant tumors in the control group. Bunse et al. demonstrated that adoptive transfer of T cells generated from C57BL/6J mice vaccinated with IDH1-R132H peptide vaccine reduced tumor growth in GL261-IDH-mutant Rag-2 knockout mice only when combined with an IDH inhibitor (BAY1436032).25 Together these studies illustrate the potential role of IDH inhibition in improving vaccine efficacy in IDH-mutant glioma.

\section{Checkpoint Blockade With IDH Inhibition}

In preclinical models, the combination of an IDH inhibitor with PD-1 blockade resulted in an increase in overall survival in mice harboring IDH-mutant gliomas compared with PD-1 blockade alone. ${ }^{25}$ In a separate study, Kadiyala et al. targeted the ligand of PD-1 by blocking PD-L1 in mice with IDH-mutant glioma. ${ }^{31}$ The authors showed that mice bearing IDH-mutant tumors treated with PD-L1 blockade + IDH inhibitor + standard-of-care therapy survived longer compared with IDH inhibitor + standard-ofcare therapy. Similarly, Friedrich et al. also inhibited PDL1 but did not directly mitigate 2HG-mediated immune suppression using an IDH inhibitor and instead used an AhR inhibitor to target 2HG-mediated immune suppression in MDMs. ${ }^{27}$ They demonstrated that the combination of an AhR inhibitor with PD-L1 blockade resulted in an increase in overall survival in mice harboring IDH-mutant gliomas compared with PD-L1 blockade alone, suggesting that reversal of the 2HG-mediated MDMs immunosuppressive phenotype augments PD-L1 blockade to promote antitumor immunity in IDH-mutant glioma. The aforementioned studies all highlight the potential importance of reversing 2HG-mediated immunosuppressive mechanisms, which may enhance the efficacy of immunomodulatory therapies such as vaccines and ICI in IDH-mutant glioma (Table 2).

\section{Discussion}

Phase I clinical data have demonstrated that IDH-mutant peptide vaccination is safe and immunogenic in humans. Despite the promising data and support for further development of this platform, several questions remain. Is the presence of $2 \mathrm{HG}$ in the tumor microenvironment of these patients counterproductive to the induction of T-cell immunity? What is the immune contexture at progressive stages? If the IDH1-R132H mutation is an early oncogenic event in gliomagenesis and possesses human leukocyte antigen-restricted immunogenicity against this ubiquitously expressed neoantigen, how do these tumor cells evade immune surveillance in the CNS? Growing evidence suggests the production of $2 \mathrm{HG}$ plays an immunosuppressive role in tumor development and recurrence.

Current vaccine and ICI therapy for IDH-mutant glioma may be hindered by $2 \mathrm{HG}$ suppressing T-cell responses both directly and indirectly. Manipulation of $2 \mathrm{HG}$ using small-molecule IDH inhibitors may augment antitumor immunity by sensitizing the TIME for optimal T-cell cytotoxicity. Once T cells are reinvigorated and/or induced to infiltrate the tumor through IDH inhibition, their activity may be mitigated by the potential resurgence of myeloidderived suppressor cells and Tregs as well as other immunosuppressive mediators such as indoleamine 2,3-dioxygenase, arginase, TGF- $\beta$, and IL- 10 . Future studies examining the role of IDH inhibition on other aspects of immune contexture are needed. Although IDH inhibition may enhance the efficacy of immune-based therapies, they may result in the opposite effect for other promising targeted therapies that rely on 2HG-generated vulnerabilities such as poly(ADP-ribose) polymerase and nicotinamide phosphoribosyltransferase inhibitors. ${ }^{32,33}$

In the context of IDH-wild-type glioma, identifying predictive biomarkers and monitoring of immune responses have been limited. For all cancers, TMB is believed to be a strong correlate with the level of preexisting antitumor immunity and responsiveness to ICI.7 In June 2020, the FDA granted approval for pembrolizumab (anti-PD1) for any solid tumor with high TMB, characterized as having $\geq 10$ mutations per megabase. ${ }^{34}$ As more patients with IDH-mutant glioma enter early-phase immunotherapy clinical trials, a concerted effort should be made to evaluate predictive biomarkers specific for IDH-mutant patients, as their responsiveness is likely different from that of IDH-wild-type tumors. Growing evidence suggests that the intratumoral topography or spatial information between immune cell types may be informative as a potential biomarker for immunotherapy. ${ }^{35}$ 


\section{Conclusions}

Malignant gliomas harboring IDH mutations represent a unique opportunity for therapeutic targeting. The IDH mutation drives a distorted, immunologically quiescent glioma TIME. This is largely driven by the production of $2 \mathrm{HG}$, an immunosuppressive molecule working in a "dual" direct and indirect fashion to dampen T-cell responses. Patients with IDH-mutant glioma are entering immunotherapy clinical trials. Immune profiling at baseline and in response to these agents may identify predictive biomarkers for this class of gliomas. Immunotherapies for IDH-mutant glioma may be augmented by abrogating 2HG-mediated immune suppression using an IDH inhibitor.

\section{Acknowledgments}

This work was supported by the Jenny Fund (www. thejennyfund.org) and A Shot For Life (www.ashotforlife.org).

\section{References}

1. Louis DN, Perry A, Reifenberger G, et al. The 2016 World Health Organization Classification of Tumors of the Central Nervous System: a summary. Acta Neuropathol. 2016;131(6): 803-820.

2. Yan H, Parsons DW, Jin G, et al. IDH1 and IDH2 mutations in gliomas. N Engl J Med. 2009;360(8):765-773.

3. Hendriks LEL, Henon C, Auclin E, et al. Outcome of patients with non-small cell lung cancer and brain metastases treated with checkpoint inhibitors. J Thorac Oncol. 2019;14(7):12441254.

4. Tawbi HA, Forsyth PA, Algazi A, et al. Combined nivolumab and ipilimumab in melanoma metastatic to the brain. $N$ Engl J Med. 2018;379(8):722-730.

5. Platten M, Bunse L, Wick A, et al. A vaccine targeting mutant IDH1 in newly diagnosed glioma. Nature. 2021; 592(7854):463-468.

6. Zhao J, Chen AX, Gartrell RD, et al. Immune and genomic correlates of response to anti-PD-1 immunotherapy in glioblastoma. Nat Med. 2019;25(3):462-469.

7. Yarchoan M, Hopkins A, Jaffee EM. Tumor mutational burden and response rate to PD-1 inhibition. N Engl J Med. 2017; 377(25):2500-2501.

8. van Thuijl HF, Mazor T, Johnson BE, et al. Evolution of DNA repair defects during malignant progression of low-grade gliomas after temozolomide treatment. Acta Neuropathol. 2015;129(4):597-607.

9. Johnson BE, Mazor T, Hong C, et al. Mutational analysis reveals the origin and therapy-driven evolution of recurrent glioma. Science. 2014;343(6167):189-193.

10. Barthel FP, Johnson KC, Varn FS, et al. Longitudinal molecular trajectories of diffuse glioma in adults. Nature. 2019; 576(7785):112-120.

11. Quail DF, Joyce JA. The microenvironmental landscape of brain tumors. Cancer Cell. 2017;31(3):326-341.

12. Ott M, Prins RM, Heimberger AB. The immune landscape of common CNS malignancies: implications for immunotherapy. Nat Rev Clin Oncol. 2021;18(11):729-744.

13. Thorsson V, Gibbs DL, Brown SD, et al. The immune landscape of cancer. Immunity. 2018;48(4):812-830.e14.

14. Richardson LG, Choi BD, Curry WT. (R)-2-hydroxyglutarate drives immune quiescence in the tumor microenvironment of IDH-mutant gliomas. Transl Cancer Res. 2019;8(2)(suppl 2): S167-S170.

15. Kohanbash G, Carrera DA, Shrivastav S, et al. Isocitrate dehydrogenase mutations suppress STAT1 and CD8+ T cell accumulation in gliomas. J Clin Invest. 2017;127(4):1425-1437.
16. Berghoff AS, Kiesel B, Widhalm G, et al. Correlation of immune phenotype with IDH mutation in diffuse glioma. Neuro Oncol. 2017;19(11):1460-1468.

17. Richardson LG, Nieman LT, Stemmer-Rachamimov AO, et al. IDH-mutant gliomas harbor fewer regulatory $\mathrm{T}$ cells in humans and mice. OncoImmunology. 2020;9(1):1806662.

18. Mathewson ND, Ashenberg O, Tirosh I, et al. Inhibitory CD161 receptor identified in glioma-infiltrating $\mathrm{T}$ cells by single-cell analysis. Cell. 2021;184(5):1281-1298.e26.

19. Friebel E, Kapolou K, Unger S, et al. Single-cell mapping of human brain cancer reveals tumor-specific instruction of tissue-invading leukocytes. Cell. 2020;181(7):1626-1642.e20.

20. Klemm F, Maas RR, Bowman RL, et al. Interrogation of the microenvironmental landscape in brain tumors reveals disease-specific alterations of immune cells. Cell. 2020;181(7): 1643-1660.e17.

21. Hambardzumyan D, Gutmann DH, Kettenmann H. The role of microglia and macrophages in glioma maintenance and progression. Nat Neurosci. 2016;19(1):20-27.

22. Amankulor NM, Kim Y, Arora S, et al. Mutant IDH1 regulates the tumor-associated immune system in gliomas. Genes Dev. 2017;31(8):774-786.

23. Danaher P, Warren S, Lu R, et al. Pan-cancer adaptive immune resistance as defined by the Tumor Inflammation Signature (TIS): results from The Cancer Genome Atlas (TCGA). J Immunother Cancer. 2018;6(1):63.

24. Han S, Liu Y, Cai SJ, et al. IDH mutation in glioma: molecular mechanisms and potential therapeutic targets. $\mathrm{Br} \mathrm{J} \mathrm{Can-}$ cer. 2020;122(11):1580-1589.

25. Bunse L, Pusch S, Bunse T, et al. Suppression of antitumor T cell immunity by the oncometabolite (R)-2-hydroxyglutarate. Nat Med.2018;24(8):1192-1203.

26. Zhang X, Rao A, Sette P, et al. IDH mutant gliomas escape natural killer cell immune surveillance by downregulation of NKG2D ligand expression. Neuro Oncol. 2016;18(10):14021412.

27. Friedrich M, Sankowski R, Bunse L, et al. Tryptophan metabolism drives dynamic immunosuppressive myeloid states in IDH-mutant gliomas. Nature Cancer. 2021;2(7):723-740.

28. Stein EM, DiNardo CD, Pollyea DA, et al. Enasidenib in mutant $I D H 2$ relapsed or refractory acute myeloid leukemia. Blood. 2017;130(6):722-731.

29. Zhu AX, Macarulla T, Javle MM, et al. Final results from ClarIDHy, a global, phase III, randomized, double-blind study of ivosidenib (IVO) versus placebo (PBO) in patients (pts) with previously treated cholangiocarcinoma (CCA) and an isocitrate dehydrogenase 1 (IDH1) mutation. J Clin Oncol. 2021;39(3)(suppl):266.

30. Lu M, Mellinghoff IK, Diaz A, et al. Inhibiting IDH mutations in low-grade glioma alters cellular function and the immune environment. Presented at: American Association for Cancer Research Annual Meeting; June 22-24, 2020. Virtual meeting. Session II.

31. Kadiyala P, Carney SV, Gauss JC, et al. Inhibition of 2-hydroxyglutarate elicits metabolic reprogramming and mutant IDH1 glioma immunity in mice. J Clin Invest. 2021;131(4): 139542.

32. Tateishi K, Wakimoto H, Iafrate AJ, et al. Extreme vulnerability of IDH1 mutant cancers to NAD+ depletion. Cancer Cell. 2015;28(6):773-784.

33. Nagashima H, Lee CK, Tateishi K, et al. Poly(ADP-ribose) glycohydrolase inhibition sequesters $\mathrm{NAD}^{+}$to potentiate the metabolic lethality of alkylating chemotherapy in IDHmutant tumor cells. Cancer Discov. 2020;10(11):1672-1689.

34. Khasraw M, Walsh KM, Heimberger AB, Ashley DM. What is the burden of proof for tumor mutational burden in gliomas? Neuro Oncol. 2020;23(1):17-22.

35. Lu S, Stein JE, Rimm DL, et al. Comparison of biomarker modalities for predicting response to PD-1/PD-L1 checkpoint 
blockade: a systematic review and meta-analysis. JAMA Oncol. 2019;5(8):1195-1204.

\section{Disclosures}

The authors report no conflict of interest concerning the materials or methods used in this study or the findings specified in this paper.

\section{Author Contributions}

Conception and design: Curry, Richardson, Choi. Acquisition of data: Richardson, Miller, Kitagawa. Analysis and interpretation of data: Curry, Richardson, Miller, Kitagawa, Choi. Drafting the article: Richardson. Critically revising the article: all authors. Reviewed submitted version of manuscript: Curry, Richardson, Miller, Wakimoto, Choi. Administrative/technical/material support: Richardson, Kitagawa. Study supervision: Curry, Choi.

\section{Correspondence}

William T. Curry: Massachusetts General Hospital, Boston, MA. wcurry@mgh.harvard.edu. 